\title{
Novel variants in GNAI3 associated with auriculocondylar syndrome strengthen a common dominant negative effect
}

\author{
Vanessa L Romanelli Tavares ${ }^{1,11}$, Christopher T Gordon ${ }^{2,11}$, Roseli M Zechi-Ceide ${ }^{3}$, Nancy Mizue Kokitsu-Nakata ${ }^{3}$, \\ Norine Voisin ${ }^{2}$, Tiong Y Tan ${ }^{4}$, Andrew A Heggie ${ }^{5}$, Siulan Vendramini-Pittoli ${ }^{3}$, Evan J Propst ${ }^{6}$, Blake C Papsin ${ }^{6}$, \\ Tatiana T Torres ${ }^{7}$, Henk Buermans ${ }^{8}$, Luciane Portas Capelo ${ }^{1,9}$, Johan T den Dunnen ${ }^{8}$, Maria L Guion-Almeida ${ }^{3}$, \\ Stanislas Lyonnet ${ }^{2,10}$, Jeanne Amiel ${ }^{2,10}$ and Maria Rita Passos-Bueno ${ }^{\star, 1}$
}

Auriculocondylar syndrome is a rare craniofacial disorder comprising core features of micrognathia, condyle dysplasia and question mark ear. Causative variants have been identified in PLCB4, GNAI3 and EDN1, which are predicted to function within the EDN1-EDNRA pathway during early pharyngeal arch patterning. To date, two GNAI3 variants in three families have been reported. Here we report three novel GNAI3 variants, one segregating with affected members in a family previously linked to 1p21.1-q23.3 and two de novo variants in simplex cases. Two variants occur in known functional motifs, the G1 and G4 boxes, and the third variant is one amino acid outside of the G1 box. Structural modeling shows that all five altered GNAI3 residues identified to date cluster in a region involved in GDP/GTP binding. We hypothesize that all GNA/3 variants lead to dominant negative effects.

European Journal of Human Genetics (2015) 23, 481-485; doi:10.1038/ejhg.2014.132; published online 16 July 2014

\section{INTRODUCTION}

Auriculocondylar syndrome (ACS, OMIM 602483 and 614669) is a rare disorder of the first and second pharyngeal arches, mainly characterized by micrognathia, agenesis or hypoplasia of the mandibular condyle and a typical auricular malformation known as a question mark ear (QME). Other frequently associated malformations include abnormal palate, microstomia, full cheeks, glossoptosis, respiratory distress and hearing loss. A wide range of inter- and intrafamilial clinical variability is observed in ACS. ${ }^{1}$

We previously mapped the first ACS locus to 1p21.1-q23.3 (ACS1) in a large Brazilian family that was initially described by GuionAlmeida et al. ${ }^{3}$ Genetic heterogeneity was also suggested as affected members of two other families were not associated with this locus. ${ }^{2,4}$ Rieder et $a l^{5}$ subsequently showed that variants in phospholipase C beta 4 (PLCB4), at 20p12.2, and in guanine nucleotide binding protein ( $\mathrm{G}$ protein) alpha-inhibiting activity polypeptide 3 (GNAI3), located within the 1p21.1-q23.3 candidate interval, are responsible for most ACS cases. GNAI3 and PLCB4 are predicted to be signaling molecules of the endothelin 1 (EDN1)-endothelin receptor type A (EDNRA) pathway, which is important for patterning of the pharyngeal arches in animal models. ${ }^{1,5}$ The involvement of this pathway in ACS was recently confirmed by the finding of EDN1 variants in ACS and in isolated QMEs (OMIM 612798). ${ }^{6}$
Thus far only two GNAI3 variants, c.118G $>$ C and c.141C $>$ A (predicted consequence p.(Gly40Arg) and p.(Ser47Arg), respectively) in three unrelated familial cases have been reported. ${ }^{5,7}$ No de novo variants have been described. Both variants are located within the G1 box, one of the five conserved motifs (G1-G5) involved in binding guanosine diphosphate (GDP)/guanosine triphosphate (GTP) in the catalytic domain of G-alpha proteins and RAS family members. ${ }^{8}$ It is unclear whether these variants have a gain-of-function ${ }^{5}$ or dominant negative effect. ${ }^{7} \mathrm{~A}$ larger number of cases is necessary to elucidate these questions.

Here we report the molecular analysis of GNAI3 in the original ACS1 Brazilian family linked to $1 \mathrm{p} 21.1-\mathrm{q} 23.3^{2}$ and in two sporadic ACS cases without previous genetic investigations. ${ }^{9}$ We describe a novel heterozygous variant in GNAI3 in each case. These variants are predicted to interfere with GDP/GTP binding, supporting a dominant negative mode of action for GNAI3 variants in ACS.

\section{MATERIALS AND METHODS}

Patients and DNA samples

Approval for this study was obtained from the Biosciences Institute Research Ethics Committee of the University of São Paulo (USP) and from the Comité de Protection des Personnes Ile-de-France II. Clinical descriptions have been previously reported for family ACS1, referred to as F2 in Masotti et al,,$^{2,3}$ and the sporadic case in Propst et al, ${ }^{9}$ hereafter referred to as Sp1. Sporadic case 2

${ }^{1}$ Centro de Pesquisas do Genoma Humano e Células Tronco, Departamento de Genetica e Biologia Evolutiva, Instituto de Biociências, Universidade de São Paulo, São Paulo, Brazil; 2INSERM U1163, Université Paris Descartes-Sorbonne Paris Cité, Institut Imagine, Paris, France; ${ }^{3}$ Department of Clinical Genetics, Hospital of Rehabilitation of Craniofacial Anomalies, University of São Paulo (HRCA-USP), Bauru, Brazil; 'Victorian Clinical Genetics Services, Murdoch Children's Research Institute, Royal Children's Hospital, and Department of Paediatrics, University of Melbourne, Melbourne, Victoria, Australia; ${ }^{5}$ Department of Plastic and Maxillofacial Surgery, Royal Children's Hospital, Melbourne, Victoria, Australia; ${ }^{6}$ Department of Otolaryngology - Head \& Neck Surgery, The Hospital for Sick Children, Toronto, Ontario, Canada; ${ }^{7}$ Institute of Biosciences, University of São Paulo, São Paulo, Brazil; ${ }^{8}$ Leiden Genome Technology Center, Leiden University Medical Center, Leiden, The Netherlands; ${ }^{9}$ Instituto de Ciência e Tecnologia, Universidade Federal de São Paulo, São José dos Campos, Brazil; ${ }^{10}$ Département de Génétique, Hôpital Necker-Enfants Malades AP-HP, Paris, France

*Correspondence: Dr MR Passos-Bueno, Departamento de Genetica e Biologia Evolutiva, Instituto de Biociências, Universidade de São Paulo, Rua do Matão, 277, sala 200, São Paulo 05508-090, Brazil. Tel: +55 113091 9910; Fax: +55 113091 7419; E-mail: passos@ib.usp.br

${ }^{11}$ These authors contributed equally to this work.

Received 27 February 2014; revised 3 June 2014; accepted 6 June 2014; published online 16 July 2014 
(Sp2; Supplementary Figure S1), initially referred for hemifacial microsomia, presented with micrognathia, microstomia, bifid uvula, right lateral tongue polyp, full cheeks, a right QME and normal left ear, conductive hearing loss, severe obstructive sleep apnea and systolic murmur. His development is within normal limits. He had normal full spine X-ray, renal ultrasound scan and SNP microarray (HumanCoreExome-12 v1.0) (Illumina, San Diego, CA, USA). A clinical summary of all cases is in Supplementary Table S1. Methods for extraction of genomic DNA, Sanger sequencing and microsatellite analysis, and programs used for analysis of variants are provided in Supplementary Information. Variants were submitted to the GNAI3 gene variant database (http://www.LOVD.nl/GNAI3).

\section{RESULTS}

\section{Family ACS1}

A novel heterozygous, predicted missense variant was identified in exon 7 of GNAI3: c.805A > T; p.(Asn269Tyr) (RefSeq: NM_006496.3) (Figure 1a). Except for one non-penetrant individual (II-7), the variant segregates with the ACS phenotype. Reconstruction of previously published haplotypes of the chromosome 1 linkage region ${ }^{2}$ along with the GNAI3 genotypes showed that individual II-7 shares only a proximal region of the at-risk haplotype (Supplementary Figure S2).

\section{Sp1}

Sequencing of GNAI3 revealed a heterozygous variant in exon 2: c. $134 \mathrm{G}>\mathrm{T}$ predicted to give the missense change p.(Gly45Val) (Figure $1 \mathrm{~b}$ ). The variant was de novo in the patient as it was not present in parental DNA.

\section{Sp2}

A de novo, heterozygous, predicted missense variant was identified in exon 2 of GNAI3: c.143C>A; p.(Thr48Asn) (Figure 1c).
Variants affecting Asn269, Gly45 or Thr48 of GNAI3 are absent from dbSNP137 and the Exome Variant Server. Variants affecting Asn269 are also not present in 275 ethnically matched control Brazilian samples. To confirm that the $\mathrm{Sp} 1$ and $\mathrm{Sp} 2$ variants were de novo, polymorphic microsatellites were tested in each family; all microsatellites (11/11 in Sp1 and 8/8 in Sp2) were consistent with paternity.

All three GNAI3 variants are predicted to disrupt a nucleotide and amino acid highly conserved in vertebrates (Figure 2), suggesting important roles for these residues in protein function. Each aminoacid change was predicted as probably damaging by PolyPhen- 2 and damaging by SIFT.

GDP/GTP binding in the catalytic domain of GNAI3 involves five small motifs, the G1-G5 boxes. The p.(Gly45Val) and p.(Thr48Asn) variants fall within and one amino acid outside of the G1 box (residues 40-47), respectively. The p.(Asn269Tyr) variant falls within the G4 box (Figure 2a-c). Mapping of the amino acids that show variants in ACS to a published GNAI3 crystal structure ${ }^{10}$ indicates that the side chain of Asn269 forms hydrogen bonds that contact GDP and the G1 box, whereas the backbone of Gly45 and the backbone and side chain of Thr48 also form hydrogen bonds with GDP (Figure 3). Variant of these residues may therefore directly compromise binding of GNAI3 to GDP/GTP.

\section{DISCUSSION}

In this study, we demonstrate that the GNAI3 variant, p.(Asn269Tyr), is the likely cause of ACS in the original ACS1 family, thus confirming our previous linkage analysis. ${ }^{2}$ The non-penetrant individual (II-7) in this family is consistent with the incomplete penetrance observed in

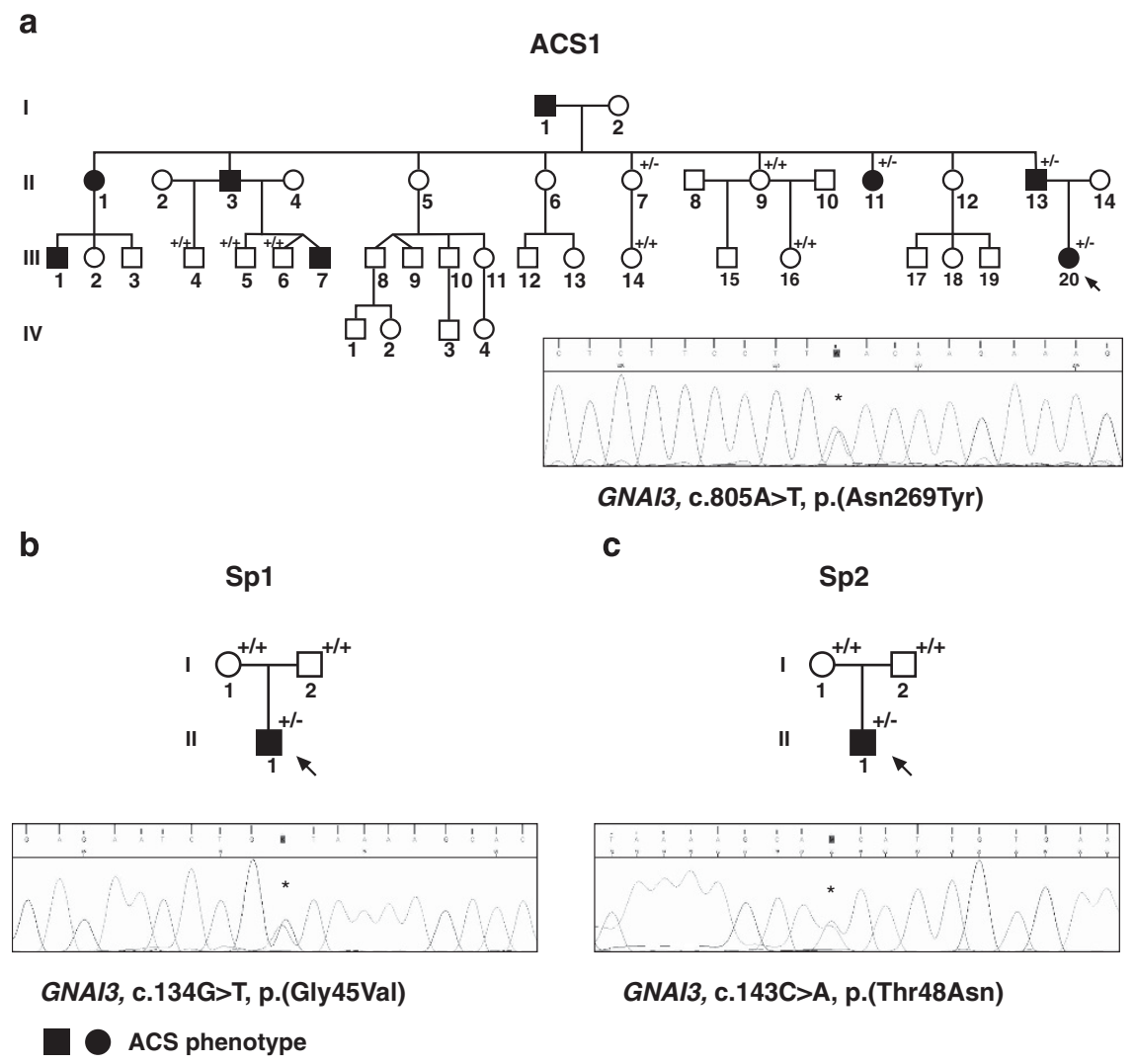

Figure 1 Identification of novel heterozygous, predicted missense GNAI3 variants. (a) GNA/3 c.805A $>$ T variant in the ACS1 family. (b) GNA/3 c.134G $>$ T variant in Sp1. (c) GNAI3 c.143C > A variant in Sp2. Wild-type allele is indicated by a plus ( +) sign; the allelic variant is represented by a minus ( - ) sign in the pedigree and indicated with an asterisk in the chromatogram; the arrows indicate proband. 
a

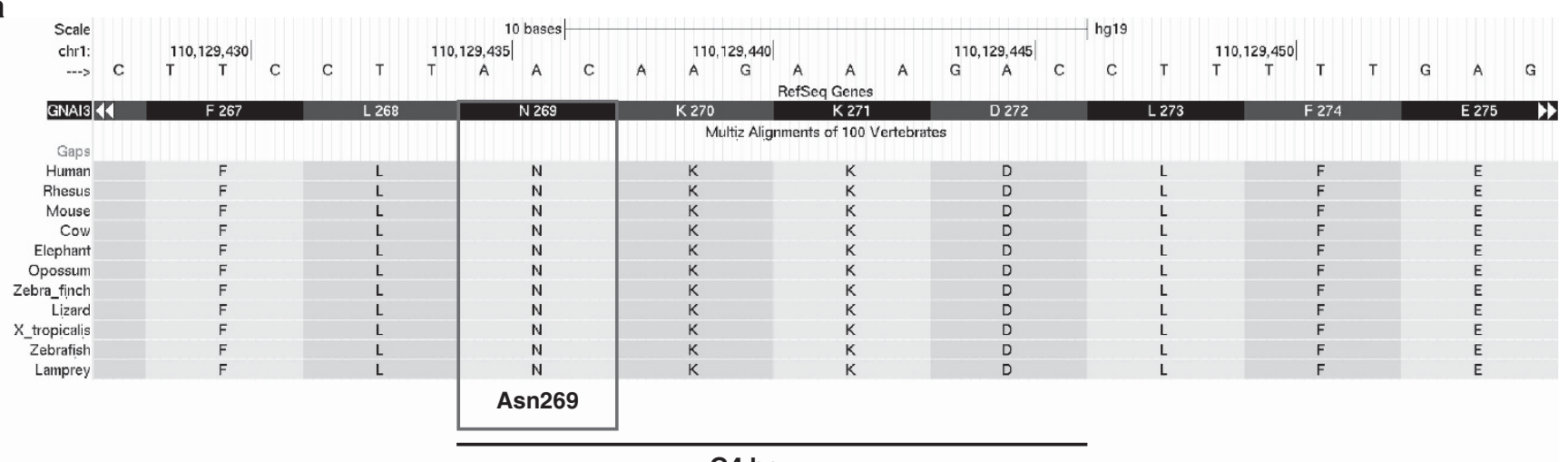

G4 box

b

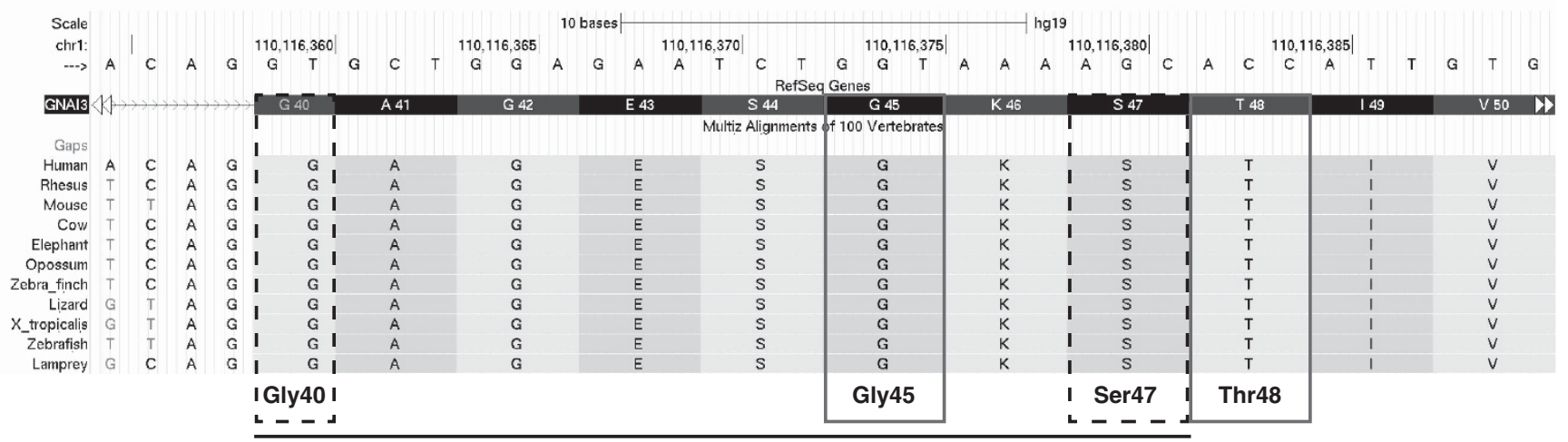

G1 box

C

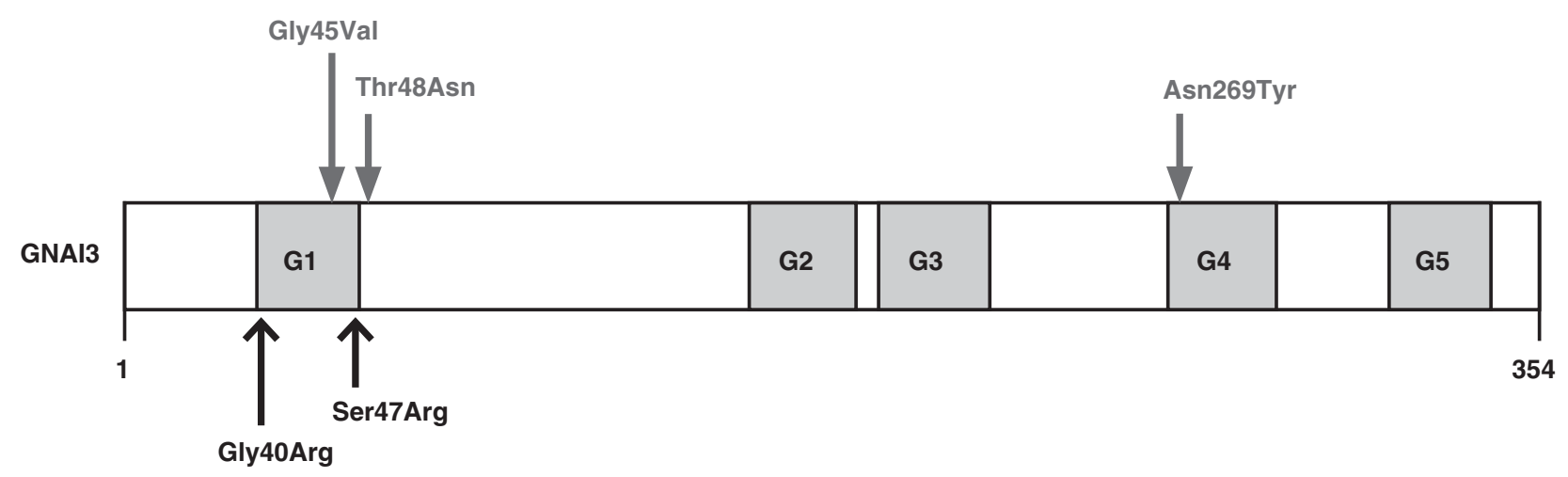

Figure 2 Conservation and location of GNAI3 residues altered in ACS. (a, b) UCSC screenshot displaying conservation of the GNAI3 G1 and G4 boxes (underlined) throughout vertebrates. The position of the variants identified here and previously reported variants are indicated by full and dashed boxes, respectively. (c) Schematic of GNAI3 showing conserved domains as described in the CDD; G1-G5 boxes are depicted in gray; previously described variants are indicated with arrows below the schema; the present variants are indicated with full arrows above the schema.

another family with a GNAI3 variant. ${ }^{5}$ We also report the first de novo variants in GNAI3 in two simplex ACS cases, Sp1 and Sp2. Our study brings the total of ACS cases harboring a GNAI3 variant to six, comprised of five different variants.

G-alpha proteins and RAS family members share a structural domain involved in GDP/GTP binding that is composed of the G1-G5 boxes. ${ }^{8}$ The two previously reported GNAI3 variants fall within the G1 box (at Gly40 and Ser47), as does the p.(Gly45Val) variant identified here in Sp1, whereas the p.(Thr48Asn) variant of Sp2 falls one amino acid outside of the G1 box. p.(Asn269Tyr) is the first variant located within the G4 box. All three of the variant residues identified here (Gly45, Thr48 and Asn269), along with Ser47, are predicted to form hydrogen bonds with GDP in the GNAI3 crystal structure. Supporting the significance of the p.(Asn269Tyr) variant, contact between hydrogen bonds of the G4 box residues of GTPase superfamily members and the guanine ring of GDP/GTP are known to confer specificity to GTP over ATP and provide stabilizing interactions with G1 box residues. $^{11}$ Although it was speculated that p.(Gly40Arg) modified the conformation of a portion of the protein that 


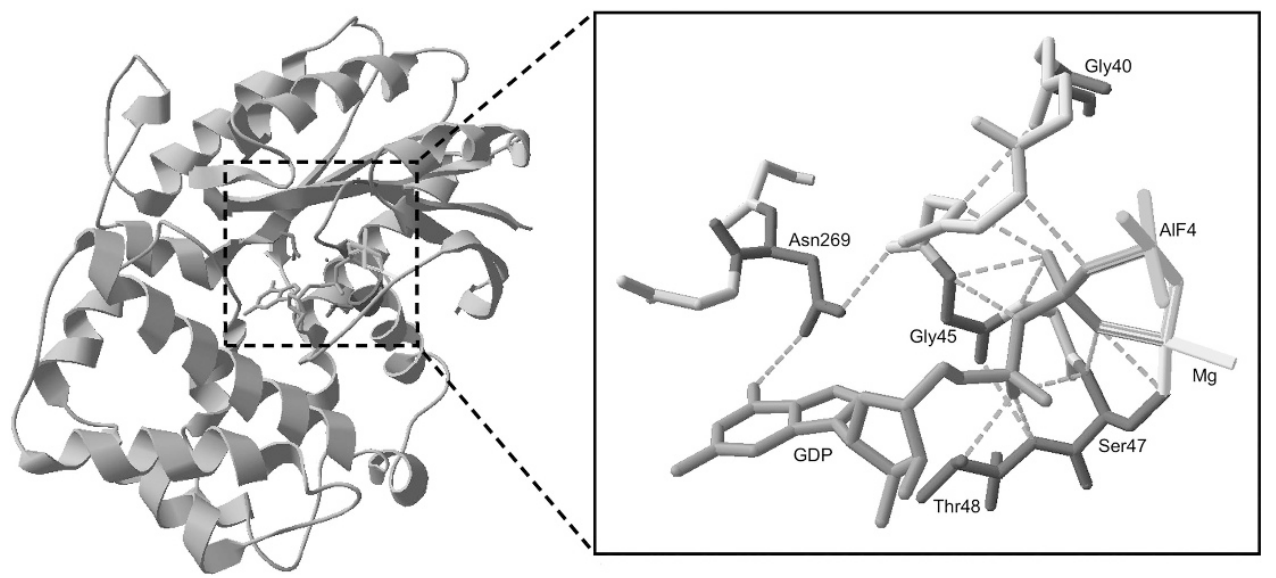

Figure 3 Structure of the GNAI3 protein (PDB ID: 2ODE) and the positions of residues affected in ACS. To the left is a view of the entire protein in ribbon mode. To the right is a magnified view of selected regions surrounding the GDP molecule, in stick mode. The five amino acids that show variants in ACS are in pink (Gly40, Gly45, Ser47, Thr48 and Asn269). For clarity, side chains are only shown for these five. Hydrogen bonds are shown as green dotted lines. $\mathrm{AlF}_{4}$ (aluminum tetrafluoride) is a substitute for the third phosphate of GTP. Mg, magnesium.

interacts with downstream effectors, resulting in a gain-of-function protein, ${ }^{5}$ we suggest rather that all GNAI3 variants may disrupt GDP/GTP binding (directly or indirectly) without disrupting the overall structure of the protein, thereby resulting in dominant negative effects, perhaps via sequestration of GNAI3's cognate beta-gamma $G$ protein subunits or $G$ protein-coupled receptor, as has been shown for other G-alpha proteins. ${ }^{12}$ Supporting this idea, the equivalent variant to GNAI3 p.(Asn269Tyr) in the G4 box of HRAS (p.Asn116Tyr) shows a dominant negative effect, inhibiting GTP binding activity and proliferation, and causing induction of apoptosis in human cancer cell lines. ${ }^{13,14}$ Similarly, the previously published p.(Ser47Arg) GNAI3 variant is predicted to be a dominant negative, based on the dominant negative action of other $G$ proteins and RAS family members with a variant of the equivalent residue. ${ }^{7}$ Although GNAI3 belongs to the inhibitory class of G-alpha proteins, originally described for their ability to inhibit adenylyl cyclase, it has been reported that activation of $\mathrm{G}$ protein heterotrimers containing GNAI3 leads to inhibitory or stimulatory responses, depending on the downstream effector: adenylyl cyclase or phospholipase C, respectively. ${ }^{15}$ Supporting the idea that GNAI3 and PLCB4 variants have a similar negative effect on the EDN1-EDNRA-DLX pathway, expression of DLX5 and DLX6 was reduced in mandibular osteoblasts of ACS patients mutated for GNAI3 or PLCB4. ${ }^{5}$ Finally, several deletions that remove GNAI3 have been reported in the DECIPHER database; of the eight cases with phenotypes listed, auricular malformations are not mentioned, supporting the idea that the ACS GNAI3 variants are not haploinsufficient alleles.

Sp1 and Sp2 have conductive hearing loss, which in Sp1 was associated with fusion of the malleus and incus, ${ }^{9}$ and one GNAI3variant individual in the ACS1 family presented with sensorineural hearing loss. ${ }^{3}$ Hearing loss was reported in both members (conductive in one case and unspecified in the other) of a family harboring a GNAI3 variant. ${ }^{5,16}$ Interestingly, zebrafish with variants in components of the endothelin pathway display fusion of some jaw cartilage elements. ${ }^{1}$ In addition, targeted deletion of Gnai3 in mice results in rib and vertebral fusions ${ }^{17}$ and in defects in cochlear hair cells. ${ }^{18}$ Collectively these findings suggest independent roles for GNAI3 in the development of multiple skeletal elements and in the inner ear, suggesting the possibility of conductive and/or sensorineural hearing loss in GNAI3-associated ACS.
In conclusion, here we have described three new ACS-associated variants in GNAI3. We suggest that these and previously described GNAI3 variants interfere directly or indirectly with GDP/GTP binding, leading to dominant negative effects. Our analysis indicates that interaction with GDP/GTP will be a strong predictor of pathogenicity for future ACS-associated GNAI3 variants.

\section{CONFLICT OF INTEREST}

The authors declare no conflict of interest.

\section{ACKNOWLEDGEMENTS}

JA and SL were supported by funding from CRANIRARE, from a Université Paris Descartes-Sorbonne Paris Cité Pole de Recherche et d'Enseignement Supérieur grant (project number SPC/JFG/2013-031) and from the Agence Nationale de la Recherche (project EvoDevoMut 2010). MRPB is funded by CEPID/FAPESP and CNPq. TYT is supported by a Training Fellowship (\#607431) from the National Health and Medical Research Council of Australia. The authors are grateful to Dr Débora Romeo Bertola for comments on this manuscript and to Regina Bueno for figure editing.

1 Clouthier DE, Passos-Bueno MR, Tavares ALP, Lyonnet S, Amiel J, Gordon CT: Understanding the basis of auriculocondylar syndrome: insights from human, mouse and zebrafish genetic studies. Am J Med Genet C Semin Med Genet 2013; 163 306-317.

2 Masotti C, Oliveira KG, Poerner F et al: Auriculo-condylar syndrome: mapping of a first locus and evidence for genetic heterogeneity. Eur J Hum Genet 2008; 16: 145-152.

3 Guion-Almeida ML, Zeichi-Ceide RM, Vendramini S, Kokitsu-Nakata NM Auriculo-condylar syndrome: additional patients. Am J Med Genet 2002; 112: 209-214.

4 Kokitsu-Nakata NM, Zeichi-Ceide RM, Vendramini-Pittoli S, Tavares VLR, PassosBueno MR, Guion-Almeida ML: Auriculo-condylar syndrome. Confronting a diagnostic challenge. Am J Med Genet A 2012; 158A: 59-65.

5 Rieder MJ, Green GE, Park SS et al: A human homeotic transformation resulting from mutations in PLCB4 and GNAI3 causes auriculocondylar syndrome. Am J Hum Genet 2012; 90: 907-914.

6 Gordon CT, Petit F, Kroisel PM et al: Mutations in endothelin 1 cause recessive auriculocondylar syndrome and dominant isolated question-mark ears. Am J Hum Genet 2013; 93: 1118-1125.

7 Gordon CT, Vuillot A, Marlin S et al: Heterogeneity of mutation mechanisms and modes of inheritance in auriculocondylar syndrome. J Med Genet 2013; 50: 174-186.

8 Wennerberg K, Rossman KL, Der CJ: The Ras superfamily at a glance. J Cell Sci 2005; 118: 843-846.

9 Propst EJ, Ngan BY, Mount RJ et al: Ossicular fusion and cholesteatoma in auriculocondylar syndrome: in vivo evidence of arrest of embryogenesis. Laryngoscope 2013; 123: 528-532.

10 Soundararajan M, Willard FS, Kimple AJ et al: Structural diversity in the RGS domain and its interaction with heterotrimeric $\mathrm{G}$ protein alpha-subunits. Proc Natl Acad Sci USA 2008; 105: 6457-6462. 
11 Colicelli J: Human RAS superfamily proteins and related GTPases. SCi STKE 2004; 2004: RE13.

12 Barren B, Artemyev NO: Review mechanisms of dominant negative G-protein a subunits. J Neurosci Res 2007; 3514: 3505-3514.

13 Shichinohe T, Senmaru N, Furuuchi K et al: Suppression of pancreatic cancer by the dominant negative ras mutant, N116Y. J Surg Res 1996; 66: 125-130.

14 Clanton DJ, Hattori S, Shih TY: Mutations of the ras gene product p21 that abolish guanine nucleotide binding. Proc Natl Acad Sci USA 1986; 83: 5076-5080.
15 Hunt TW, Carroll RC, Peraltas G, Heterotrimeric G: Proteins containing Gai3 regulate multiple effector enzymes in the same cell. J Biol Chem 1994; 269: 29565-29570.

16 Erlich MS, Cunningham ML, Hudgins L: Transmission of the dysgnathia complex from mother to daughter. Am J Med Genet 2000; 95: 269-274.

17 Plummer NW, Spicher K, Malphurs J et al: Development of the mammalian axial skeleton requires signaling through the Gai subfamily of heterotrimeric $\mathrm{G}$ proteins. Proc Natl Acad Sci USA 2012; 109: 21366-21371.

18 Ezan J, Lasvaux L, Gezer A et al: Primary cilium migration depends on G-protein signaling control of subapical cytoskeleton. Nat Cell Biol 2013; 15: 1107-1115.

Supplementary Information accompanies this paper on European Journal of Human Genetics website (http://www.nature.com/ejhg) 